\title{
Acerca da Detecção Automática de Silhuetas de Manchas de Óleo no Mar
}

\author{
Mauricio de P. Rodrigues ${ }^{1}$, Bianca O. B. de Carvalho ${ }^{2}$, Wesley L. Passos ${ }^{1}$, Sergio L. Netto ${ }^{1,2}$, Eduardo A. B. da \\ Silva $^{1,2}$, Marcel M. Mendes ${ }^{1}$, Levi W. de Resende Filho ${ }^{1,3}$, Lucas V. Vargas ${ }^{1}$ e Alessandro J. Peixoto ${ }^{1,2}$.
}

Resumo-A detecção precoce de derramamentos de óleo é fundamental para medidas de resposta rápida. $O$ uso de imagens adquiridas por drones, combinado com técnicas de visão computacional e aprendizado profundo, apresenta oportunidades de automação das atividades de vigilância de derramamentos de óleo. No entanto, o treinamento adequado de modelos capazes de detectar objetos e regiões de interesse em imagens geralmente requer um grande conjunto de dados anotados. Neste trabalho, apresentamos um método semi-automático para acelerar o processo de anotação de imagens de manchas de óleo, gerando silhuetas no nível do pixel, baseado nos algoritmos de segmentação Mean Shift e Simple Linear Iterative Clustering. Como resultado, construímos um banco de imagens anotadas com silhuetas de derramamentos de óleo usando a metodologia proposta.

Palavras-Chave-Derramamento de Óleo, Mean Shift, Segmentação de Imagens, Silhuetas, Simple Linear Iterative Clustering (SLIC), Superpixel.

Abstract-Early detection of oil spills is paramount for quick response measures. The use of images acquired by UAVs (unmanned aerial vehicles), combined with computer vision techniques and deep-learning, presents automation opportunities to oil spill surveillance activities. However, proper training of models capable of detecting objects and region of interest in images often requires a large annotated dataset. In this work, we present a semi-automatic method to accelerate the process of annotating oil spill images generating silhouettes at pixel level based on the mean shift and simple linear iterative clustering segmentation algorithms. As a result, we built an annotated database of oil spill images by using the proposed methodology.

Keywords-Image Segmentation, Mean Shift, Oil spill, Silhouettes, Simple Linear Iterative Clustering (SLIC), Superpixel.

\section{INTRODUÇÃO}

O derramamento de óleo em operações off-shore frequentemente acarreta prejuízos de ordens ambiental, econômica e social, sem contar o dano à imagem das empresas envolvidas no incidente. Em 2019, o vazamento causado por um navio petroleiro foi considerado o maior desastre ambiental da história no litoral brasileiro em termos de extensão, atingindo mais de 2.100 quilômetros de extensão e causando prejuízo da ordem de bilhões de reais, segundo estimativas do IBAMA [1].

Com o objetivo de minimizar os impactos causados por esses incidentes, as ações de resposta aos derramamentos de óleo devem ser tomadas no tempo mais curto possível. Devido ao fato do petróleo possuir a tendência de se espalhar e volatilizar, diversas técnicas usadas para a mitigação dos efeitos dos vazamentos perdem eficácia com o tempo [2].

${ }^{1}$ PEE/COPPE, Universidade Federal do Rio de Janeiro, Rio de Janeiro, RJ, Brasil. ${ }^{2}$ DEL/Poli, Universidade Federal do Rio de Janeiro, Rio de Janeiro, RJ, Brasil. ${ }^{3}$ PROFICAM, Universidade Federal de Ouro Preto e Instituto Tecnológico Vale. e-mails:\{mauricio.rodrigues, bianca.carvalho, wesley.passos, sergioln, eduardo\}@smt.ufrj.br, marcel.mendes@ coppe.ufrj.br, levi.filho@aluno.ufop.edu.br, vares.lucas@gmail.com, jacoud@poli.ufrj.br.
Desta forma, a detecção precoce de derramamentos de óleo é essencial para a efetividade destas ações de resposta.

Tipicamente, a análise e detecção de derramamentos de óleo são realizadas por observadores humanos especialistas [2], que são capazes de estimar o volume do óleo derramado assim como o tempo transcorrido desde o início do incidente. No entanto, evolução das tecnologias de sensoriamento remoto, visão computacional e aprendizado de máquina, combinadas ao avanço de capacidade computacional, permitem a automação dessas atividades, reduzindo a dependência humana na detecção e, dessa forma, acelerando o processo de remediação da causa raiz do problema e contenção do óleo derramado.

\section{A. Métodos de detecção de manchas de óleo no mar}

De acordo com o trabalho em [3], os tipos de sensores mais utilizados na detecção de derramamentos de óleo são:

- Sensores ópticos:

- Espectro visível;

- Infravermelho;

- Próximo ao Infravermelho;

- Ultravioleta.

- Sensores de fluorescência induzida por laser.

- Sensores na faixa de microondas:

\section{- Sensores passivos;}

- Radares.

Atualmente, imagens obtidas por radares de abertura sintética (synthetic aperture radar, SAR), a partir de satélites, são as dominantes no monitoramento de operações off-shore. No entanto, este tipo de imagem apresenta algumas desvantagens tais como: baixa resolução, baixa frequência de atualização e custo elevado. Dessa forma, a utilização de drones equipados com câmeras que atuam nos espectros visível e/ou infravermelho apresentam uma proposta promissora para suportes tático e operacional [4]. Isto acontece porque drones portando câmeras, por voarem a baixa altitude, capturam informações de cor e não tem sua atuação atrapalhada por nuvens. Além disso, a sua grande facilidade de posicionamento e flexibilidade de uso também contribuem para reduzir o tempo de detecção.

O desenvolvimento de técnicas de detecção automática de manchas de óleo no mar usando imagens requer a disponibilidade de um número grande e diverso o suficiente de imagens de mar com manchas de óleo para que modelos eficientes e efetivos possam ser treinados. Assim sendo, a construção de um banco de imagens onde as manchas de óleo estão indicadas e rotuladas, é etapa essencial no desenvolvimento deste tipo de técnicas. Até o momento da publicação deste trabalho, não se tem conhecimento de uma base pública de dados anotada com imagens aéreas de derramamentos de óleo obtidas por 
câmeras convencionais. É importante notar que a construção de um banco de imagens que seja diverso o suficiente para ser útil no treinamento de modelos de detecção automática é uma tarefa laboriosa. Este trabalho se encaixa neste contexto, e seu objetivo é propor um método que acelere o processo de anotação de manchas de óleo em imagens, culminando na criação de uma base de dados anotada de imagens de manchas de óleo.

Para melhor apresentar nossas principais contribuições técnicas, este artigo é organizado da seguinte forma: Na Seção II são apresentados os trabalhos de detecção de derramamento de óleo em operações off-shore utilizando técnicas de aprendizado profundo em imagens obtidas por câmeras convencionais ou por SAR. Como não há uma base de imagens de derramamento de óleo, obtidas por câmeras convencionais, que esteja anotada e seja pública, este trabalho traz como contribuição a definição de um procedimento detalhado para construção de uma base deste tipo. Na Seção III é apresentada uma metodologia para anotação, em múltiplas classes, das imagens no nível do pixel, utilizando técnicas de segmentação de imagem para acelerar o processo. Na Seção IV são apresentados exemplos de imagens anotadas pela metodologia proposta. E, por fim, na Seção V, são apresentadas as conclusões deste trabalho e propostas de trabalhos futuros.

\section{TRABALHOS RELACIONADOS}

Atualmente, há diversos trabalhos relacionados à detecção automática de derramamento de óleo em mares e oceanos, com prevalência dos que utilizam imagens capturadas por satélite. Embora a detecção através de imagens de satélite venha sendo largamente utilizada, este método apresenta algumas desvantagens, tais como: maiores atrasos na captura das imagens, dificuldade de distinção entre os variados tipos de óleo, possibilidade de oclusão das imagens devido a condições atmosféricas desfavoráveis, entre outros.

Em [4] é realizada uma revisão dos principais métodos utilizados para medir a espessura de derramamentos de óleo. Os autores do trabalho verificam que a categorização do óleo utilizando apenas dados no espectro visível é pouco eficaz devido a fatores como condições climáticas, variação de ângulo de incidência e intensidade da luz solar. No entanto, esta referência também ressalta que o uso de câmeras no espectro visível em conjunto com câmeras na faixa do infravermelho embarcadas em drones tem se mostrado promissor.

Nos trabalhos em [5] e [6] são utilizadas imagens coloridas adquiridas a partir de aeronaves. Em [5], a base de dados utilizada possui 250 imagens. São propostos métodos de extração de atributos de cor e textura baseadas em histogramas e transformadas wavelets, seleção dos atributos que melhor identificam variações intraclasse das manchas de óleo e, por fim, o treinamento de um modelo supervisionado baseado em máquinas de vetores-suporte (support vector machines) para detecção de óleo nas imagens. Em [6], a base utilizada possui 946 imagens onde são empregadas técnicas de aumento de dados (data augmentation) como rotação das imagens e variação na intensidade de iluminação; em seguida, é construído um modelo de detecção de objeto baseado numa region-based convolutional neural network (faster R-CNN).

No trabalho em [7] foram utilizadas 2.530 imagens capturadas a partir de satélites utilizando tecnologia SAR. Foi treinada uma rede do tipo mask region-based CNN baseada na arquitetura ResNet-101 para realizar a segmentação.

Em [8], é proposto um arcabouço de aprendizado profundo para detecção e categorização de manchas de óleo. A detecção é realizada baseada em segmentação semântica de imagens SAR obtidas por satélites. Os pixels de cada imagem são classificados como "óleo" ou "não-óleo". Após a segmentação semântica, as imagens são dividas em patches, onde cada patch contendo óleo é classificado em 12 possíveis classes, de acordo com sua forma e textura.

Em [9], são propostos dois métodos para classificar imagens como contendo ou não derramamento de óleo. O primeiro realiza segmentação baseada nos atributos de cor da imagem nos espaços $\mathrm{HSV}, \mathrm{YCbCr}$ e $\mathrm{L}^{*} \mathrm{a} * \mathrm{~b}$ utilizando o algoritmo $k$ means. Os resultados das segmentações são combinados e, com base em limiares estabelecidos, o classificador determina as regiões com mancha de óleo. O segundo método analisa o desempenho de diferentes arquiteturas de redes neurais convolucionais, onde a DenseNet-161 obtém os melhores resultados.

\section{Metodologia Proposta}

Os algoritmos de inteligência computacional, em particular os de aprendizado profundo, têm tido relevância crescente na classificação e detecção em imagens. Entretanto, a construção de modelos que apresentem bons resultados exige que o seu treinamento seja realizado em uma base de dados extensa e robusta.

Em visão computacional, quando se trata da compreensão de imagens por máquinas, há quatro categorias principais de tarefas, a saber: (1) classificação de imagens [10], (2) detecção de objetos [11], (3) segmentação semântica [12] e (4) segmentação por instância [13]. Neste trabalho, optouse por realizar a segmentação semântica, que é equivalente a classificar cada pixel de uma imagem. A segmentação semântica possui algumas vantagens quando comparada a outros métodos de detecção, tais como permitir a estimação da área afetada pelo derramamento e a detecção mais refinada dos contornos das regiões de interesse.

Para construir um modelo capaz de realizar segmentação semântica, é necessário que as imagens do conjunto de dados de treinamento estejam anotadas no nível do pixel, isto é, à cada pixel da imagem será atribuído um rótulo que corresponde à classe à qual aquele pixel pertence.

Dentro deste contexto, o processo de criação da base anotada proposta foi dividido nas seguintes etapas:

- Aquisição de um conjunto de imagens de derramamento de óleo;

- Definição das classes;

- Segmentação das imagens;

- Anotação dos segmentos.

\section{A. Definição das classes}

Derramamentos de óleo no mar podem apresentar aspectos visuais distintos dependendo de diversos fatores como o tipo de óleo, a quantidade derramada e o tempo transcorrido desde o início do incidente. Visando padronizar o método de classificação do óleo derramado e auxiliar na estimação da área afetada, a União Europeia estabeleceu o Acordo de Bonn 
TABELA I: Acordo de Bonn para Código de Aparência de Óleo.

\begin{tabular}{l|c|c}
\hline Aparência & Espessura $(\mu m)$ & Litros por $\mathrm{km}^{2}$ \\
\hline Code 1: Filme (cinza) & $0,04-0,3$ & $40-300$ \\
Code 2: Arco-íris & $0,30-5$ & $300-5.000$ \\
Code 3: Metálica & $5-50$ & $5.000-50.000$ \\
Code 4: Cores descontínuas & $50-200$ & $50.000-200.000$ \\
Code 5: Cores contínuas & $>200$ & $>200.000$ \\
\hline
\end{tabular}

definindo um Código de Aparência de Óleo (Bonn Agreement Oil Appearance Code) [14], conforme a Tabela I.

O processo de marcação aqui proposto considera, além das classes de óleo caracterizadas na Tabela I, outras classes de interesse, conforme definido na Tabela II.

TABELA II: Classes utilizadas na anotação dos segmentos

\begin{tabular}{l|c}
\hline Classe & Cor \\
\hline Óleo preateado & cinza claro \\
Óleo arco-íris & roxo \\
Óleo metálico & cinza escuro \\
Óleo emulsificado & laranja \\
Água limpa & azul \\
Embarcação & amarelo \\
Plataforma & marrom \\
Indefinida & branco \\
Fronteira & verde \\
\hline
\end{tabular}

\section{B. Segmentação das imagens}

A anotação de imagens no nível do pixel é, por si só, uma tarefa demorada, entediante e sujeita a erros. Quando se trata de derramamento de óleo em mares e oceanos, a atividade tornase ainda mais complexa devido às características irregulares e de dispersão inerentes aos fluídos. Além disso, segundo [4] a correta classificação de manchas de óleo na superfície do mar a partir de imagens obtidas por sensores ópticos pode ser dificultada pelas condições nas quais as imagens foram capturadas. Portanto, para auxiliar e acelerar o processo de análise e anotação das imagens, a proposta deste trabalho é que as imagens sejam pré-segmentadas antes do processo de anotação.

A segmentação de imagens é o processo de agrupar pixels de uma imagem em múltiplas regiões e classificá-los de forma que pixels de um mesmo agrupamento compartilhem certas características como, por exemplo, cor, intensidade e localização espacial. Como resultado, a segmentação tende a produzir uma representação simplificada e informativa da imagem. Por exemplo, a segmentação pode ser utilizada para localizar objetos e suas respectivas silhuetas nas imagens.

Existem diversos algoritmos de segmentação de imagens [15], que podem ser classificados em: (1) métodos baseados em estimação de densidade como, por exemplo, o mean shift [16] e o quick shift [17]; (2) métodos baseados em grafos, como o normalized cuts [18] e o de Felzenszwalb e Huttenlocher [19]; e (3) métodos baseados em agrupamentos (clustering) como o k-means e o simple linear iterative clustering (SLIC) [20].

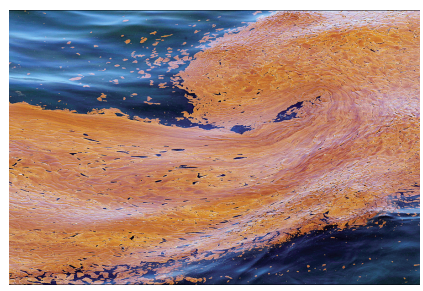

(a)

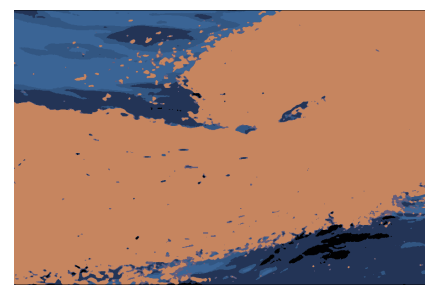

(b)
Fig. 1: Imagem de óleo emulsificado: (a) Original; (b) Segmentada com MS em RGB.

Devido à facilidade de implementação associada a bons resultados de desempenho, os métodos de segmentação escolhidos neste trabalho são o mean shift e o SLIC, brevemente descritos a seguir.

1) Mean Shift (MS): é um método não-paramétrico de estimativa de densidade baseado em kernel. Em segmentação de imagens, este método utiliza o espaço formado pelos atributos espaciais e de cor de cada pixel da imagem para estimar os máximos locais (modas) das funções densidade de probabilidade neste espaço. O MS é caracterizado por (I) o tipo de kernel $k(\|\mathbf{x}\|)$ utilizado; e (II) sua largura de $h$. No MS, o kernel é radial, simétrico e com integral igual a um. Exemplos típicos de kernel são o uniforme, o gaussiano e o de Epanechinikov.

Para $K$ pontos $\mathbf{x}_{k}, k=1, \ldots, K$ no espaço d-dimensional de atributos, o MS é implementado aplicando, a cada ponto $\mathbf{x}_{k}$, a Equação (1) recursivamente até a convergência da série $\mathbf{x}_{k}^{(n)}, n=0,1,2, \ldots$ abaixo:

$$
\mathbf{x}_{k}^{(n+1)}=\frac{\sum_{\left\|\mathbf{x}_{i}-\mathbf{x}_{k}^{(n)}\right\|<h} \mathbf{x}_{i} g\left(\left\|\frac{\mathbf{x}_{k}^{(n)}-\mathbf{x}_{i}}{h}\right\|^{2}\right)}{\sum_{\left\|\mathbf{x}_{i}-\mathbf{x}_{k}^{(n)}\right\|<h} g\left(\left\|\frac{\mathbf{x}_{k}^{(n)}-\mathbf{x}_{i}}{h}\right\|^{2}\right)}
$$

onde $\mathbf{x}_{k}^{0}=\mathbf{x}_{k}, h$ é a largura do kernel $k(u)$ e $g(u)=-k^{\prime}(u)$. Todos os $\mathbf{x}_{k}$ para os quais as respectivas séries $\mathbf{x}_{k}^{(n)}, n=$ $0,1,2, \ldots$ convergem para um mesmo ponto são considerados como pertencendo à mesma região.

No MS, não há controle sobre a quantidade de segmentos que o algoritmo irá produzir. No entanto, este valor é determinado indiretamente pela largura $h$ do kernel. Valores pequenos de $h$ tendem produzir imagens com maior número de segmentos. Essa característica é particularmente importante para imagens com manchas de óleo muito finas, pois a pequena variação de cor entre a mancha de óleo fina e a água limpa pode fazer com que o algoritmo as classifiquem dentro de um mesmo segmento. Por outro lado, para imagens com óleo emulsificado, como visto na Figura 1, é preferível utilizar valores mais elevados de $h$, a fim de evitar uma imagem supersegmentada.

O resultado da segmentação, é influenciado pelo espaço de cor utilizado. Neste trabalho, utiliza-se os espaços RGB e o HSV para que a anotação seja feita sobre o resultado que apresenta a melhor qualidade de segmentação. 


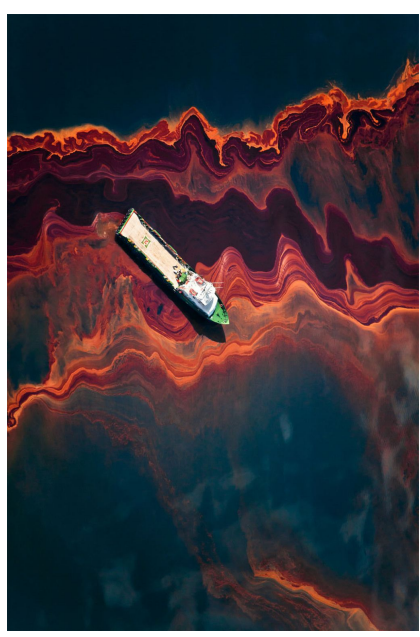

(a)

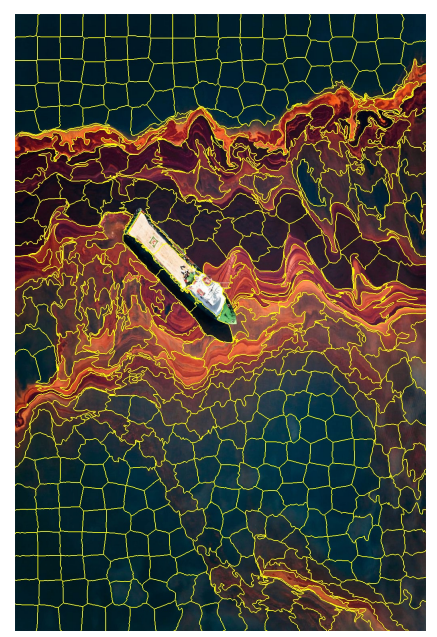

(b)

Fig. 2: Supersegmentação de derramamento de óleo: (a) Imagem original; (b) Resultado da segmentação com SLIC.

2) Simple Linear Iterative Clustering (SLIC): é um método não-supervisionado de segmentação de imagens em superpixels. Algoritmos de superpixels [20] agrupam pixels que compartilham determinadas características em regiões atômicas visualmente significativas, reduzindo, assim, a complexidade de processamento subsequente. Tipicamente, estes agrupamentos são realizados com base em métricas de similaridade de cor e proximidade espacial, gerando imagens supersegmentadas como a da Figura 2b.

No SLIC, o parâmetro de entrada $k$ determina a quantidade aproximada de superpixels desejados. Cada pixel é representado por sua cor no espaço CIELAB [21] $\left[\begin{array}{lll}l & a & b\end{array}\right]$ e por sua posição $\left[\begin{array}{ll}x & y\end{array}\right]$ no plano da imagem, formando assim o espaço 5D $\left[\begin{array}{lllll}l & a & b & x & y\end{array}\right]$. Cada pixel $x_{i}$ na imagem fará parte do superpixel $S_{k}$, com centróide $c_{k}$, de modo que a distância euclidiana no espaço $\left[\begin{array}{lllll}l & a & b & x & y\end{array}\right]$ entre $x_{i}$ e $c_{k}$ seja mínima. Assim, a medida de distância entre o pixel $p_{i}$ e o centróide $c_{k}$ é definida como:

$$
D=\sqrt{\left(\frac{d_{c}}{N_{c}}\right)^{2}+\left(\frac{d_{s}}{N_{s}}\right)^{2}},
$$

onde

$$
\begin{aligned}
& d_{c}=\sqrt{\left(l_{i}-l_{k}\right)^{2}+\left(a_{i}-a_{k}\right)^{2}+\left(b_{i}-b_{k}\right)^{2}}, \\
& d_{s}=\sqrt{\left(x_{i}-x_{k}\right)^{2}+\left(y_{i}-y_{k}\right)^{2}} .
\end{aligned}
$$

Para combinar as distâncias nos domínios de cor e espacial em uma única medida de distância, é necessário normalizá-las por suas respectivas distâncias máximas, $N_{c}$ e $N_{s}$, com relação a um dado cluster.

\section{Anotação dos segmentos}

Cada imagem do conjunto de dados foi submetida inicialmente ao algoritmo MS, onde a largura do kernel é ajustada pelo operador com o objetivo de obter uma segmentação satisfatória. Nas imagens com óleo emulsificado, o contraste de cor entre o óleo e a água era grande o suficiente para que

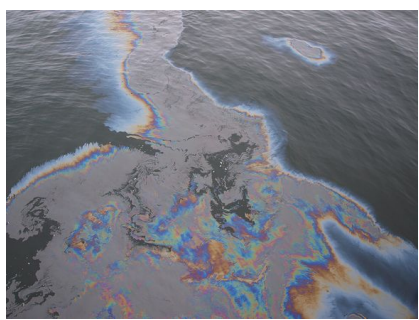

(a)

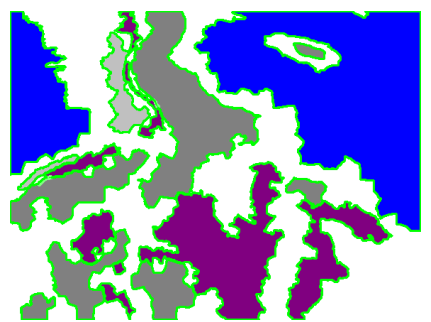

(b)
Fig. 3: Exemplo de marcação proposta com região indefinida: (a) Imagem original; (b) Marcação efetuada com região indefinida indicada em branco com fronteira verde.

o MS pudesse agrupar os pixels em suas respectivas regiões de forma adequada, como ilustrado na Figura 1b.

No entanto, em imagens que apresentam camadas finas de óleo, ou onde as manchas de óleo apresentam aspecto muito difuso, os resultados obtidos pelo MS podem não ser satisfatórios. Nesses casos utilizou-se o SLIC para segmentar a imagem em superpixels de tamanho adequado.

Após a segmentação das imagens, foi realizado o processo de anotação manual em cada um dos segmentos, atribuindolhes uma das classes descritas na Tabela II.

Mesmo após cuidadoso ajuste nos parâmetros em ambos os algoritmos de segmentação, algumas regiões das imagens segmentadas ainda são de difícil classificação. Isto ocorre devido a uma série de fatores como, por exemplo, condições meteorológicas desfavoráveis, reflexo da luz solar na superfície da água e manchas de óleo difusas ou muito finas que se confundem com o próprio mar. Notou-se, também, que há grande ocorrência de erro de classificação de pixels próximos às regiões de fronteiras entre classes. A fim de evitar que o classificador seja treinado a partir de anotações imprecisas, definiu-se uma classe chamada indefinida. Esta classe é utilizada para anotar os segmentos que demarcam regiões consideradas ambíguas. Desta forma, os pixels anotados com a classe indefinida podem ser ignorados durante as fases de treino e/ou avaliação do classificador.

Na Figura 3a é exibida imagem de derramamento de óleo com camadas nas categorias: óleo prateado, óleo arco-íris, óleo metálico e água. Na Figura $3 \mathrm{~b}$ a imagem foi anotada com as respectivas classes, no entanto, algumas regiões foram de difícil classificação. Para essas regiões a classe indefinida foi utilizada.

\section{Resultados}

A anotação no nível do pixel é uma tarefa exaustiva quando feita sem auxílio de ferramentas que acelerem o processo de marcação. A utilização de algoritmos de segmentação tornou possível a marcação de agrupamentos representativos de pixels no lugar de classificá-los individualmente. Com isso, o tempo de marcação foi significativamente reduzido. Ao todo foram anotadas 213 imagens, dentre as quais 108 continham manchas de óleo e 105 não continham manchas de óleo.

Em imagens que possuem manchas de óleo emulsificado o MS geralmente apresenta resultados satisfatórios, pois, apesar de ser mais custoso que o SLIC, gera segmentos maiores e mais fáceis de anotar manualmente. Por outro lado, em imagens com manchas de óleo mais finas, o SLIC se mostrou 

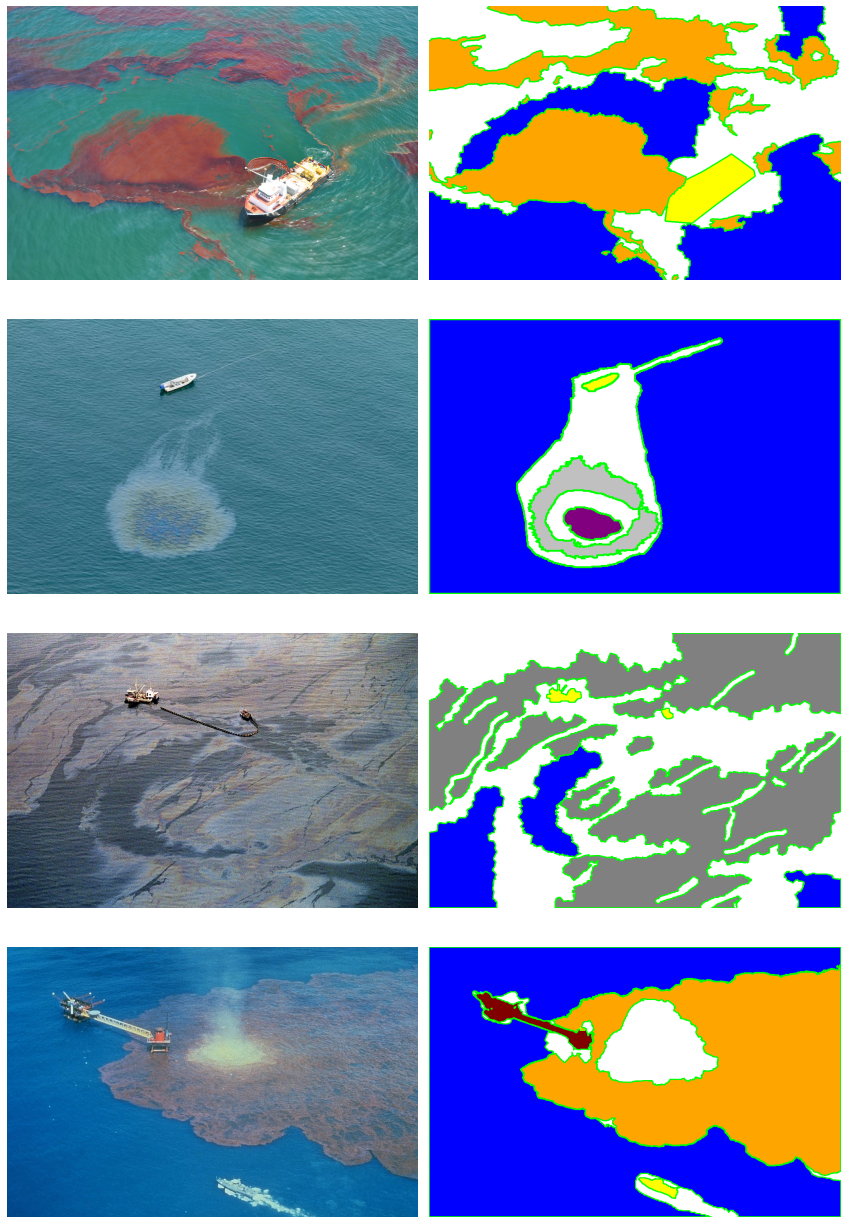

Fig. 4: Exemplos de imagens anotadas com o método proposto.

mais apropriado, pois, por ser computacionalmente menos complexo, permite ajustes no parâmetro de maneira mais ágil, além de garantir maior coerência espacial que o MS.

$\mathrm{Na}$ Figura 4, apresentam-se alguns exemplos de imagens de derramamento de óleo anotadas no nível do pixel através da abordagem proposta. Mais exemplos de imagens anotadas podem ser encontradas em https://www.smt.ufrj.br/ eduardo/oilspill/.

\section{CONCLUSÕES}

Neste trabalho, apresentaram-se: (I) uma metodologia para auxiliar e acelerar o processo de marcação de imagens de derramamento de óleo no nível do pixel em múltiplas classes; (II) uma base de dados anotada de imagens áreas obtidas por câmeras convencionais de diversos eventos de derramamento de óleo em mares e oceanos. A marcação de imagens no nível do pixel é uma tarefa demorada e sujeita a erros. No entanto, esse processo possui a vantagem de rotular cada pixel de uma imagem, sendo assim adequado para detectar precisamente objetos e regiões de interesse em uma imagem. Como trabalhos futuros, sugere-se a construção de modelos de detecção baseados em redes neurais convolucionais (CNN) que sejam capazes de realizar a segmentação semântica das manchas de derramamento de óleo utilizando a base de dados criada neste trabalho.

\section{AgRADECIMENTOS}

Os autores agradecem ao Bureau of Safety and Environmental Enforcement por ceder algumas fotos de casos reais.

\section{REFERÊNCIAS}

[1] "Prejuízo com vazamento de óleo chegará aos bilhões, diz Ibama," Valor Econômico, Nov 2019. [Online]. Available: https://valor.globo.com/brasil/noticia/2019/11/04/prejuizo-comvazamento-de-oleo-chegara-aos-bilhoes-diz-ibama.ghtml

[2] I. Leifer, W. J. Lehr, D. Simecek-Beatty, E. Bradley, R. Clark, P. Dennison, Y. Hu, S. Matheson, C. E. Jones, B. Holt et al., "State of the art satellite and airborne marine oil spill remote sensing: Application to the BP deepwater horizon oil spill,' Remote Sensing of Environment, vol. 124, pp. 185-209, 2012.

[3] M. Fingas, "The challenges of remotely measuring oil slick thickness," Remote Sensing, vol. 10, no. 2, 2018. [Online]. Available: https://www.mdpi.com/2072-4292/10/2/319

[4] M. Fingas and C. E. Brown, "A review of oil spill remote sensing," Sensors, vol. 18, no. 1, p. 91, 2018.

[5] T. Chen and S. Lu, "Subcategory-aware feature selection and SVM optimization for automatic aerial image-based oil spill inspection," IEEE Transactions on Geoscience and Remote Sensing, vol. 55, no. 9, pp. 5264-5273, 2017.

[6] Z. Jiao, G. Jia, and Y. Cai, "A new approach to oil spill detection that combines deep learning with unmanned aerial vehicles," Computers \& Industrial Engineering, vol. 135, pp. 1300-1311, 2019.

[7] S. T. Yekeen, A.-L. Balogun, and K. B. W. Yusof, "A novel deep learning instance segmentation model for automated marine oil spill detection," ISPRS Journal of Photogrammetry and Remote Sensing, vol. 167, pp. 190-200, 2020.

[8] F. M. Bianchi, M. M. Espeseth, and N. Borch, "Large-scale detection and categorization of oil spills from SAR images with deep learning," Remote Sensing, vol. 12, no. 14, p. 2260, 2020.

[9] M. Mendes, L. Resende Filho, L. Vargas, and A. Jacoud, "Visão computacional e redes neurais convolucionais aplicadas à detecção de vazamentos de Óleo," in Congresso Brasileiro de Automática, 2020, pp. $1-8$.

[10] A. Krizhevsky, I. Sutskever, and G. E. Hinton, "Imagenet classification with deep convolutional neural networks," in Advances in Neural Information Processing Systems, 2012, pp. 1097-1105.

[11] S. Ren, K. He, R. Girshick, and J. Sun, "Faster R-CNN: Towards realtime object detection with region proposal networks," IEEE Transactions on Pattern Analysis and Machine Intelligence, vol. 39, no. 6, pp. 11371149, 2017.

[12] E. Shelhamer, J. Long, and T. Darrell, "Fully convolutional networks for semantic segmentation," IEEE Transactions on Pattern Analysis and Machine Intelligence, vol. 39, no. 4, pp. 640-651, 2017.

[13] K. He, G. Gkioxari, P. Dollar, and R. Girshick, "Mask R-CNN," IEEE Transactions on Pattern Analysis and Machine Intelligence, vol. 42, no. 2, pp. 386-397, 2018.

[14] "Bonn agreement oil appearance code (BAOAC)," Jan 2004. [Online]. Available: https://www.bonnagreement.org/site/assets/files/1081/special_on_volume calculation_20160607.docx

[15] D. Stutz, A. Hermans, and B. Leibe, "Superpixels: An evaluation of the state-of-the-art," Computer Vision and Image Understanding, vol. 166, pp. 1-27, 2018.

[16] D. Comaniciu and P. Meer, "Mean shift: A robust approach toward feature space analysis," IEEE Transactions on Pattern Analysis and Machine Intelligence, vol. 24, no. 5, pp. 603-619, 2002.

[17] A. Vedaldi and S. Soatto, "Quick shift and kernel methods for mode seeking," in European Conference on Computer Vision, 2008, pp. 705718.

[18] J. Shi and J. Malik, "Normalized cuts and image segmentation," IEEE Transactions on Pattern Analysis and Machine Intelligence, vol. 22 no. 8, pp. 888-905, 2000.

[19] P. F. Felzenszwalb and D. P. Huttenlocher, "Efficient graph-based image segmentation," International Journal of Computer Vision, vol. 59, no. 2, pp. 167-181, 2004.

[20] R. Achanta, A. Shaji, K. Smith, A. Lucchi, P. Fua, and S. Süsstrunk, "SLIC superpixels compared to state-of-the-art superpixel methods," IEEE Transactions on Pattern Analysis and Machine Intelligence, vol. 34, no. 11, pp. 2274-2282, 2012.

[21] K. McLaren, "XIII - The development of the CIE $1976\left(\mathrm{~L}^{*} \mathrm{a}^{*} \mathrm{~b}^{*}\right)$ uniform colour space and colour-difference formula," Journal of the Society of Dyers and Colourists, vol. 92, no. 9, pp. 338-341, 1976. 